\title{
Irisin Regulates Heme Oxygenase-1/ Adiponectin Axis in Perivascular Adipose Tissue and Improves Endothelial Dysfunction in Diet-Induced Obese Mice
}

\author{
Ningning Hou ${ }^{a}$ Gang Du ${ }^{a}$ Fang Han ${ }^{b}$ Jin Zhang ${ }^{c}$ Xiaotong Jiao ${ }^{a}$ Xiaodong Sun ${ }^{a}$ \\ ${ }^{a}$ Department of Endocrinology, ${ }^{b}$ Department of Pathology, Affiliated Hospital of Weifang Medical \\ University, 'Office of Academic Affairs, Weifang Medical University, Weifang, China
}

\section{Key Words}

Endothelial function • Perivascular adipose tissue Irisin • Heme oxygenase-1 • Adiponectin

\begin{abstract}
Aims: To determine whether irisin could improve endothelial dysfunction by regulating heme oxygenase-1(HO-1)/adiponectin axis in perivascular adipose tissue (PVAT) in obesity. Methods: Male C57BL/6 mice were fed with a high-fat diet (HFD) with or without irisin treatment. Endothelium-dependent vasorelaxation of the thoracic aorta with or without PVAT (PVAT+ or PVAT-) was determined. Western blot was employed to determine the levels of HO- 1 and adiponectin in PVAT. UCP-1, Cidea, and TNF- $\alpha$ gene expression in PVAT were tested by real-time PCR. Results: The presence of PVAT significantly impaired endothelial function in the HFD mice. Treatment of HFD mice with irisin significantly restored this impairment and improved endothelial function in vivo or ex vivo. Incubated aortic rings (PVAT-) with PVATderived conditioned medium (CM) from HFD mice impaired endothelial function in control mice. This impairment was prevented by incubating the aortic rings (PVAT-) from HFD mice with PVAT-derived CM from irisin. However, the beneficial effects were partly attenuated in the presence of HO-1 inhibitor and adiponectin receptor blocking peptide. Treatment of HFD mice with irisin significantly increased NO production, protein levels of $\mathrm{HO}-1$ and adiponectin, mRNA expressions of UCP-1 and Cidea, and decreased superoxide production and TNF- $\alpha$ expression in PVAT. Conclusion: Irisin improved endothelial function by modulating HO-1/ adiponectin axis in PVAT in HFD-induced obese mice. These findings suggest that regulating PVAT function may be a potential mechanism by which irisin improves endothelial function in obesity.

(C) 2017 The Author(s)

Published by S. Karger AG, Basel
\end{abstract}

\section{Introduction}

Perivascular adipose tissue (PVAT), which surrounds most blood vessels, has shown to play an important role in modulating vascular function and to be a strong independent 
predictor of atherosclerosis [1, 2]. PVAT is not only considered as structural support for the vessel but also releases several adipokines that influence vascular tone in a paracrine way $[3,4]$. Under normal conditions, healthy PVAT exerts an anti-contractile effect on vessels through both endothelium-dependent and -independent mechanisms [3, 5]. However, further studies have found that these beneficial effects of PVAT are lost in obesity due to functional changes in PVAT $[4,6]$. Our previous study demonstrated that high free fatty acids (FFA) in obesity could directly cause attenuation of the anti-contractile response of PVAT through an endothelium-dependent pathway that involves the reduced production of nitric oxide (NO) [7]. PVAT has also been demonstrated to significantly exacerbate underlying endothelial dysfunction in obese swine [8]. These results suggest PVAT loses the ability to protect the vessels through promoting endothelial dysfunction in obesity.

Irisin, a newly identified hormone secreted by myocytes, can be induced by exercise and mediates browning of white adipocytes by stimulating uncoupling protein 1 expression (UCP1) $[9,10]$. Overexpression of irisin can induce weight loss and protect against dietinduced obesity [9]. Recently, many studies demonstrated that altered circulating irisin levels were associated with endothelial function. Zhu et al [10] showed that irisin improves endothelial dysfunction in diet-induced diabetic mice. In our previous study, serum irisin levels were found to be reduced in obese patients compared with non-obese subjects and that treatment of obese mice with irisin improves endothelial dysfunction by the AMP kinase (AMPK)-endothelial NO synthase (eNOS) signaling pathway $[11,12]$. These findings suggest that irisin has the ability to improve endothelial dysfunction.

The heme oxygenase-1 (HO-1)/adiponectin axis has recently demonstrated to have beneficial effects on cardiovascular disease $[13,14]$. H0-1 is the major enzyme of heme metabolism decomposition and can be induced by various oxidative insults. Upregulation of HO-1 may induce an increase in adiponectin secretion by remodeling adipose tissue, which prevents endothelial dysfunction by its anti-oxidative and anti-inflammatory effects [14-16]. Previously, we observed that irisin improves the PVAT dysfunction from the thoracic aorta in died-induced obese mice through upregulation of the HO-1/adiponectin axis and browning of PVAT [17]. These results suggest that irisin has vascular protective effects through regulating PVAT function in obesity. However, whether irisin also have direct protective effects on endothelial function by regulating HO-1/adiponectin axis in PVAT in obesity has not been clear. Therefore, the aim of the study was to determine whether irisin improves endothelial dysfunction by regulating HO-1/adiponectin axis in PVAT in obesity.

\section{Materials and Methods}

\section{Experimental animals}

Male C57BL/6 mice (4-week-old) were purchased from Beijing Vital River Laboratory Animal Technology Company (Beijing, China). We then randomly divided all mice into three groups: a normal control (NC) group, a high fat diet (HFD) group, and an irisin (irisin) group. Mice in the NC group were fed a normal chow diet and mice in the other two groups were fed a HFD (493 kcal $100 \mathrm{~g}^{-1}$ ) [18]. Mice in the irisin group were given recombinant irisin $(0.5 \mu \mathrm{g} / \mathrm{g} /$ day; Phoenix Pharmaceuticals, Inc., Burlingame, CA, USA) by intraperitoneal injection daily $[12,14]$. The other two groups were given physiological saline as control. All the mice were housed under standard laboratory conditions with free access to food and water. The local ethics committee for animal studies approved all procedures described and the handling of mice following the committee's "Principles of Laboratory Animal Care".

\section{Intraperitoneal glucose tolerance test and Insulin tolerance test}

The mice were injected with glucose $(1.5 \mathrm{~g} / \mathrm{kg})$ by intraperitoneal injection after an overnight fasting. For insulin tolerance test, the mice were fasted for $6 \mathrm{~h}$ and injected with Humulin $(0.5 \mathrm{U} / \mathrm{kg})$, tail vein blood sampling was carried out at the indicated time intervals. Glucose and insulin levels were measured by glucometer (Johnson \& Johnson, USA) and ELISA (Uscn Life Science Inc., Wuhan, China), respectively.

\section{KARGER}




\section{Cellular Physiology Cell Physiol Biochem 2017;42:603-614 \begin{tabular}{ll|l} 
and Biochemistry Published onIIne: June U9, 2017 & $\begin{array}{l}\text { C } 2017 \text { The Author(s). Published by S. Karger AG, Basel } \\
\text { www.karger.com/cpb }\end{array}$
\end{tabular}}

Hou et al.: Irisin Improves Endothelial Dysfunction

\section{Determination of blood parameters}

After 12 weeks, the mice were anesthetized with an intraperitoneal injection of sodium pentobarbital $(50 \mathrm{mg} / \mathrm{kg})$. Blood samples were collected. Serum lipids and malondialdehyde (MDA) were determined by using colorimetric assays (Beyotime Biotechnology, Beijing, China). Serum levels of irisin, TNF- $\alpha$ and adiponectin were measured using the ELISA kits (Uscn Life Science Inc.).

Preparation of PVAT-derived conditioned medium (CM)

The PVAT from the descending thoracic aorta of the mice was dissected and incubated with DMEM for $24 \mathrm{~h}$. The medium was collected as conditioned medium (CM) [19]. In some experiments, PVAT-CM from different groups was pretreated first with palmitic acid (PA, $500 \mathrm{uM}$ ) or irisin (50 nM) and then pretreated with a varieties of inhibitors individually: HO-1 inhibitor stannous protoporphyrin (SnPP, 20 uM; Frontier Scientific, Inc., Logan, UT, USA), adiponectin receptor blocking peptide (ACRP-30 N-20, 0.4 $\mu$ g/mL; Santa Cruz Biotechnology, USA), AMPK inhibitor Compound C (20 $\mu \mathrm{M}$; Merck, Darmstadt, Germany) or eNOS inhibitor L-NAME $\left(10^{-4} \mathrm{M}\right.$; Sigma) individually for $2 \mathrm{~h}$. In brief, PVAT-CM was divided into different groups and a variety of inhibitors were individually added into the different groups.

\section{Endothelium-dependent vasorelaxation (EDV) determination}

Endothelium-dependent vasorelaxation was assayed as previously described [12, 20]. Descending thoracic aorta of mice with PVAT intact (PVAT+) or carefully dissected (PVAT-) were cut into 2-mm ring segments and suspended in Krebs-Henseleit bicarbonate buffer for measurement of endothelial function (95\% oxygen and 5\% carbon dioxide). All rings were first equilibrated, pre-contracted with $\mathrm{KCl}(60 \mathrm{mM})$. Phenylephrine $(1 \mu \mathrm{M})$ was then added to produce contraction. Either ACh $\left(10^{-9}-10^{-5} \mathrm{M}\right)$ or SNP $\left(10^{-9}-10^{-5}\right.$ $\mathrm{M})$ was then added to measure EDV or endothelium-independent vasorelaxation responses. The data were recorded by PowerLab (AD Instruments, Australia). The aortic rings were then first incubated with the HO-1 inhibitor SnPP $(20 \mu \mathrm{M})$, ACRP-30 N-20, $(0.4 \mu \mathrm{g} / \mathrm{mL})$, Compound C $(20 \mu \mathrm{M})$, and L-NAME $\left(10^{-4} \mathrm{M}\right)$ for $0.5 \mathrm{~h}$, respectively followed by determination of EDV. After each experiment, we removed the compound (SnPP, ACRP-30 N-20, Compound C or L-NAME) used before completely and then incubated the aortic ring with another one.

In some experiments, the aortic ring without PVAT from control mice was first pre-treated with CM derived from HFD mice, and the aortic ring from HFD mice was first pre-treated with CM derived from irisin mice. After incubation for $30 \mathrm{~min}$, the subsequent endothelium-dependent vasorelaxation was assayed.

\section{Ex vivo culture of aortic rings}

Mouse thoracic aortic rings with or without intact PVAT from obese mice were incubated in Dulbecco's modified Eagle's medium (Gibco, Gaithersburg, MD, USA) [21]. Irisin (50 nM) [22], SnPP (20 uM), ACRP-30 $\mathrm{N}-20(0.4 \mu \mathrm{g} / \mathrm{mL})$, Compound $\mathrm{C}(20 \mu \mathrm{M})$ and L-NAME $\left(10^{-4} \mathrm{M}\right)$ were individually added into the culture media. After incubation for $60 \mathrm{~min}$, the rings were transferred to an organ bath for measuring EDV as described in Section 2.4.

\section{Total NO measurement}

Modified Griess reaction method was used to measure NO production (nitrite/nitrate concentration) as described previously $[12,23]$. After ACh $\left(10^{-5} \mathrm{M}\right)$ induced vasorelaxation, K-H solution $(100 \mu \mathrm{L})$ taken from the organ bath was mixed with Griess reagent (100 ul). After incubation for $60 \mathrm{~min}$, the NO production was spectrophotometrically determined at $540 \mathrm{~nm}$.

Measurement of superoxide production in PVAT

Superoxide production in PVAT was determined by the lucigenin chemiluminescence method as described previously [5]. Briefly, isolated PVAT was incubated in warmed PSS for $0.5 \mathrm{~h}$ before measurement of chemiluminescence. Superoxide production was measured with $5 \mu \mathrm{M}$ lucigenin-enhanced chemiluminescence technique.

Western blot analysis for protein expression in PVAT

The extracted proteins from PVAT were subjected to sodium dodecyl sulfate-polyacrylamide gel electrophoresis. The proteins were probed with antibodies against HO-1 and adiponectin (Santa Cruz 


\section{Cellular Physiology Cell Physiol Biochem 2017;42:603-614 \begin{tabular}{ll|l} 
and Biochemistry Published onlIne: June U9, 2017 & $\begin{array}{l}\text { (c) } 2017 \text { The Author(s). Published by S. Karger AG, Basel } \\
\text { www.karger.com/cpb }\end{array}$
\end{tabular}}

Biotechnology), and then incubated with secondary antibodies. The protein levels were quantified using Image Pro-plus 6.0.

RNA extraction and $q R T-P C R$

Gene expression was measured by qRT-PCR. Briefly, total RNA from PVAT was extracted using Trizol (Invitrogen, Carlsbad, CA, USA). FNDC5, TNF- $\alpha$, Cidea, and UCP-1 RNA were isolated using a Pure Link RNA mini kit (Invitrogen). Reverse transcription was performed using a High Capacity RNA-to-cDNA Kit (Applied Biosystems, USA). qRT-PCR was performed as previously described [24]. Primer sequences were used according to our previous study [17]

\section{Statistical analysis}

Statistical analysis was performed by the statistical software package SPSS 13.0 for Microsoft windows (SPSS Inc). The analysis was performed using one-way ANOVA or two-way ANOVA, as appropriate. $\mathrm{P}<0.05$ were considered statistically significant.

\section{Results}

\section{Effects of irisin on blood parameters}

Administration of HFD in the mice induced increased body weight, visceral fat, fasting glucose levels, fasting insulin levels, and lipid levels compared with the control mice $(P$ $<0.01)$. Treatment of HFD mice with exogenous irisin could modify metabolic variables $(P$ $<0.01)$. The irisin-treated HFD mice showed an improvement of both glucose tolerance and insulin sensitivity compared to HFD mice (Fig. 1). Plasma levels of TNF- $\alpha$ and MDA increased, and adiponectin levels decreased in HFD mice compared with the control group $(P<0.01)$, which were all improved by treatment of HFD mice with exogenous irisin $(P<0.01)$ (Table 1$)$.

\section{Effects of irisin on endothelial function in mice aortas}

The ACh-induced concentration-dependent vasorelaxation was similar in both the presence and absence of PVAT in control mice (Fig. 2A); while in the HFD mice, the presence of PVAT significantly impaired endothelium-mediated vasorelaxation responses to ACh (Fig. 2B). However, treatment of HFD mice with irisin restored this impairment with a similar response to both the PVAT+ ring and the PVAT- ring $(P<0.05$, Fig. $2 \mathrm{C})$. This beneficial effect of irisin was attenuated when SnPP, API-2/triciribine or Compound C were added. As expected, the ACh-stimulated vasorelaxation in aortic rings with and without PVAT were reduced in HFD mice, indicating that endothelial function was impaired both in the presence

Table 1. Biometric and blood parameters of mice in the studied groups. Data are shown as mean \pm SD. $* \mathrm{P}<0.01$ vs. control group. ${ }^{\#} \mathrm{P}<0.01$ vs. HDF group. $\mathrm{n}=6-8$ in each group. TG, triglycerides; FFA, free fatty acids; TNF- $\alpha$, tumor necrosis factor- $\alpha$; MDA, malondialdehyde

\begin{tabular}{llll}
\hline Group & Control & HFD & HFD-Irisin \\
\hline Body weight $(\mathrm{g})$ & $30.0 \pm 1.5$ & $40.3 \pm 1.9^{*}$ & $33.2 \pm 2.6^{* \#}$ \\
Visceral fat/weight $\left(10^{-3}\right)$ & $16.9 \pm 7.3$ & $47.3 \pm 7.0^{*}$ & $36.5 \pm 3.4^{* \#}$ \\
Glucose (mg/dl) & $91.7 \pm 5.2$ & $108.1 \pm 4.7^{*}$ & $98.5 \pm 4.6^{\#}$ \\
Insulin (ng/ml) & $0.90 \pm 0.09$ & $1.87 . \pm 0.20^{*}$ & $1.52 \pm 0.22^{\#}$ \\
TG (mg/dl) & $70.20 \pm 5.41$ & $132.13 \pm 8.86^{*}$ & $109.48 \pm 11.35^{\#}$ \\
FFA (mmol/L) & $0.40 \pm 0.06$ & $1.20 \pm 0.23^{*}$ & $0.90 \pm 0.18^{\#}$ \\
TNF- $\alpha(\mathrm{pg} / \mathrm{ml})$ & $13.50 \pm 1.42$ & $40.22 \pm 4.87^{*}$ & $29.02 \pm 2.56^{\#}$ \\
MDA (umol/L) & $1.25 \pm 0.12$ & $6.63 \pm 0.76^{*}$ & $5.14 \pm 0.33^{\#}$ \\
Adiponectin (ug/ml) & $21.82 \pm 1.89$ & $12.60 \pm 1.68^{*}$ & $15.86 \pm 1.04^{\#}$ \\
Irisin (ng/ml) & $110.91 \pm 13.55$ & $81.56 \pm 11.55^{*}$ & $186.5 . \pm 17.9^{\#}$ \\
\hline
\end{tabular}




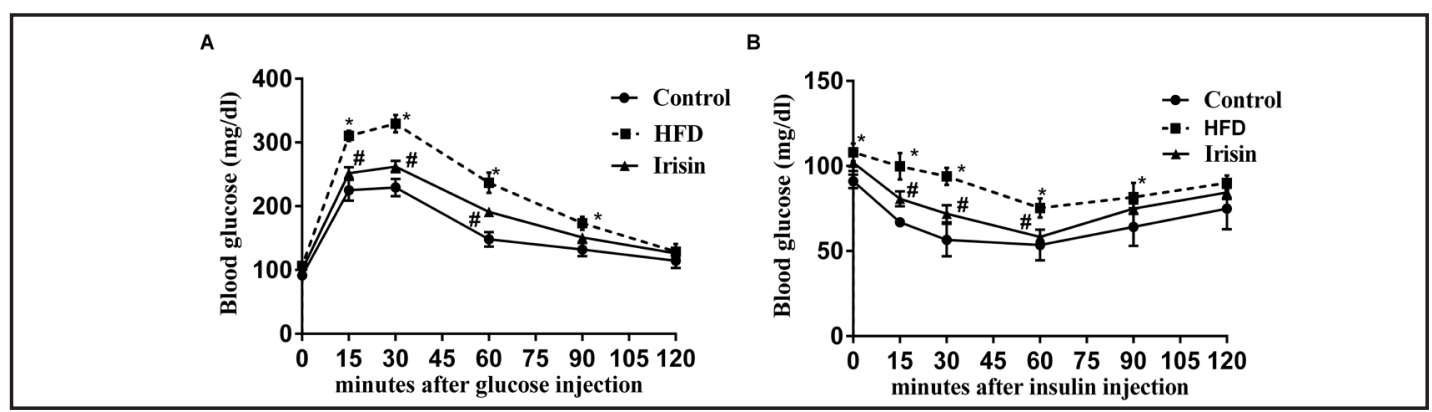

Fig. 1. Effects of irisin on intraperitoneal glucose tolerance test $(A)$ and insulin tolerance test $(B)$ in mice. $n=$ 4. ${ }^{*} \mathrm{P}<0.05$ vs. control group; ${ }^{\#} \mathrm{P}<0.05$ vs. HFD group.

Fig. 2. Effects of irisin on endothelial function in mice aortas.(A) ACh-induced concentration-dependent vasorelaxation in control mice. (B) PVAT significantly impaired endothelium-mediated vasorelaxation responses to ACh in HFD mice. ${ }^{*} \mathrm{P}<0.05$. (C) Irisin restored this impairment and the presence of SnPP, API-2/triciribine or Compound $\mathrm{C}$ partially abolished this improvement. ${ }^{*} P<$ 0.05 vs. Irisin-PVAT+. (D and E)ACh-stimulated vasorelaxation in aortic rings both with and without PVAT were lower in HFD mice compared with control mice and irisin significantly enhanced ACh-induced vasorelaxation. ${ }^{*} P<$ 0.05 vs. NC, ${ }^{\#} P<0.05$ vs. HFD. (F) Effects of irisin on endothelial function in CM-treated mice aortas. ${ }^{*} P<0.05$ vs. control, ${ }^{\#} P<0.05$ vs. HFD, ${ }^{\&} P<$ 0.05 vs. CM-Irisin. $\mathrm{n}=4-5$ in each group. NC, normal control; HFD, high fat diet, CMHFD, CM derived from PVAT of HFD; CM-Irisin, CM derived from PVAT of irisin.

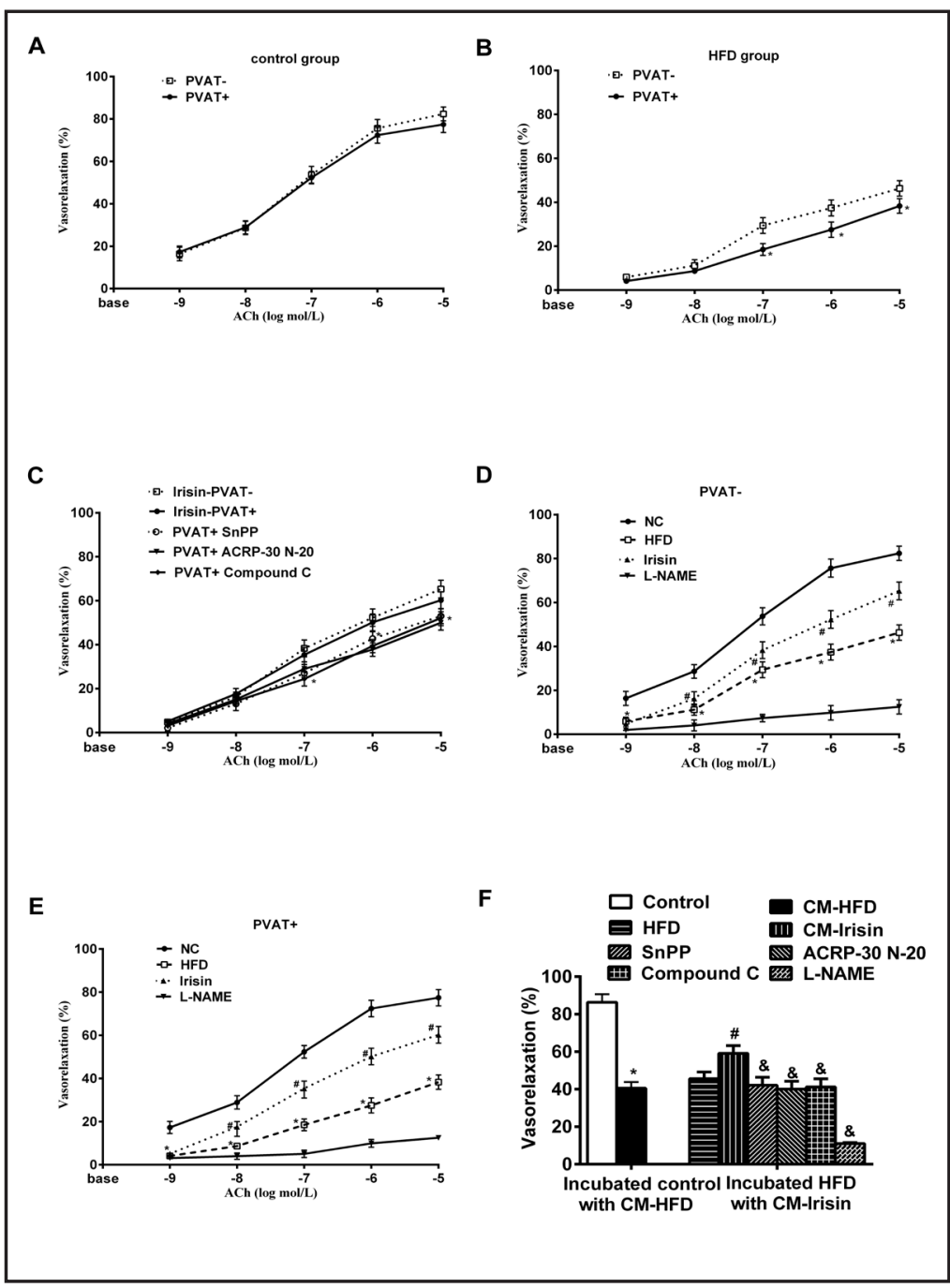

or absence of PVAT $(P<0.05$, Fig. 2D and 2E). However, treatment of HFD mice with irisin significantly improved endothelial function $(P<0.05)$. Incubation of the aortic rings with L-NAME abolished ACh-induced vasorelaxation in all groups. No difference to SNP response was observed among the rings from the three groups and PVAT did not influence the vascular responses to SNP (data not shown). 

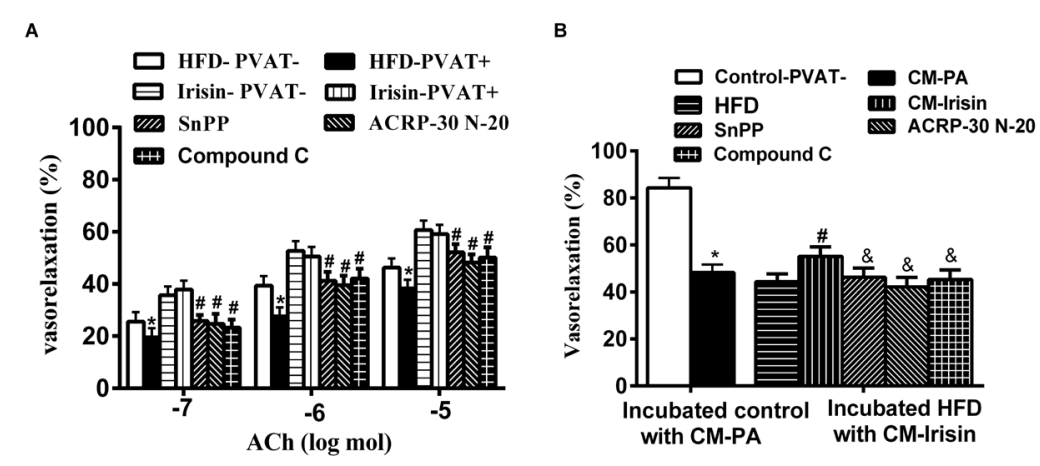

Fig. 3. Effects of irisin on endothelial function in mice aortas ex vivo. (A) PVAT significantly impaired endothelium-mediated vasorelaxation responses in HFD mice. Irisin restored this impairment and the presence of SnPP, API-2/triciribine or Compound C partially abolished this improvement. ${ }^{*} P<0.05$ vs. HFD-PVAT-; $P$ $<0.05$ vs. Irisin-PVAT-. (B) Incubated the aortic rings (PVAT-) from control mice with CM derived from PVAT of control mice treated with PA impaired vasorelaxation. However, this impairment was prevented by incubating the aortic rings (PVAT-) from HFD mice with CM derived from PVAT of HFD mice treated with irisin, and this beneficial effect was partly attenuated in the presence of SnPP, API-2/triciribine or Compound C. ${ }^{*}$ $<0.05$ vs. control PVAT-, ${ }^{\#} P<0.05$ vs. HFD, ${ }^{\circledR} P<0.05$ vs. CM-Irisin. $\mathrm{n}=4-5$ in each group. NC, normal control; HFD, high fat diet, CM-Irisin, CM derived from PVAT of HFD treated with irisin.

Fig. 4. Effect of irisin on total NO production (A) in the endothelial cells and superoxide production (B) in PVAT. $n=4-6 .{ }^{*} \mathrm{P}<0.05$ vs. control group; ${ }^{*} \mathrm{P}<0.05$ vs. HFD PVAT-; ${ }^{\&} \mathrm{P}<0.05$ vs. HFD group.

\section{Effect of irisin on endothelial function in} CM-treated mice aortas

To investigate the direct influence of PVAT on endothelial function, we prepared $\mathrm{CM}$ by incubating PVAT from mice. Aortic rings (PVAT-) from control mice incubated with CM derived from PVAT of HFD mice impaired the Ach $\left(10^{-5} \mathrm{M}\right)$-induced vasorelaxation (vasorelaxation reduced from $86.4 \%$ to $40.5 \%$ ). However, this impairment was prevented by incubating the aortic rings (PVAT-) from HFD mice with CM derived from PVAT of irisin mice $\left(10^{-5} \mathrm{M}\right.$ Ach-induced vasorelaxation enhanced from $45.6 \%$ to $59.1 \%$ ). This beneficial effect of irisin was attenuated when SnPP, API-2/triciribine or Compound C were added (Fig. 2F).

\section{Effect of irisin on endothelial function on aortic rings ex vivo}

The presence of PVAT significantly impaired endothelium-mediated relaxation responses to ACh in HFD mice. However, incubating aortic rings from HFD mice with irisin $(50 \mathrm{nM})$ ex vivo prevented this impairment. The beneficial effect of irisin on ACh-induced EDV in HFD mice was partly attenuated in the presence of SnPP, ACRP-30 N-20 or Compound C (Fig. 3A).

To investigate the direct influence of PVAT on endothelial function, CM were prepared by incubating PVAT from mice. Aortic rings (PVAT-) from control mice incubated with CM derived from PVAT of control mice treated with PA impaired the Ach $\left(10^{-5} \mathrm{M}\right)$-induced 


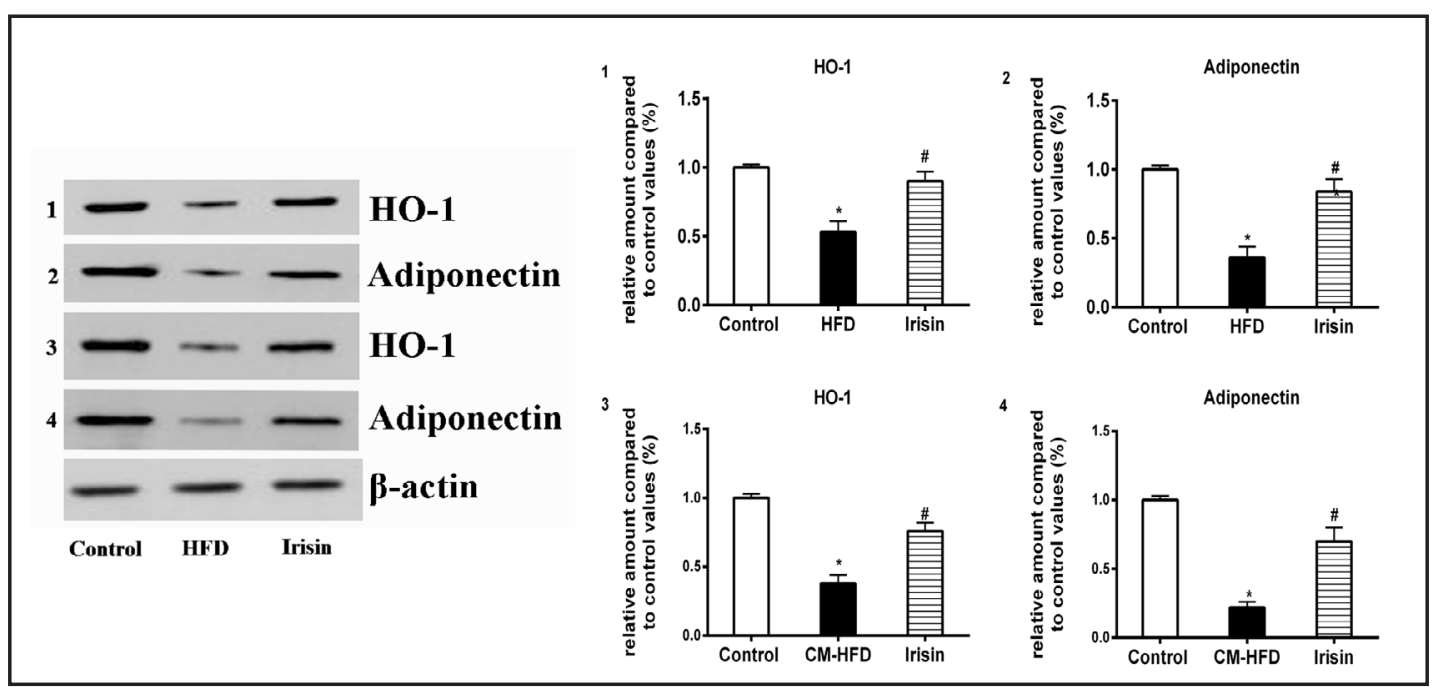

Fig. 5. Effect of irisin on HO-1/adiponectin axis in PVAT of HFD mice. (A, 1-2) HO-1 and adiponectin levels in PVAT from the HFD mice were reduced compared with those in the control mice, which were both enhanced when treating with irisin. (A, 3-4) In an ex vivo study, incubating the CM derived from PVAT of HFD mice with irisin also enhance the proteins of HO- 1 and adiponectin. ${ }^{*} P<0.05$ vs. control, ${ }^{\#} P<0.05$ vs. HFD, ${ }^{\&} P<0.05$ vs. Irisin. $\mathrm{n}=4$ in each group. $\mathrm{NC}$, normal control; HFD, high fat diet.

vasorelaxation (vasorelaxation reduced from $84.3 \%$ to $48.3 \%$ ). However, this impairment was prevented by incubating the aortic rings (PVAT-) from HFD mice with CM derived from PVAT of irisin treated mice $\left(10^{-5} \mathrm{M}\right.$ ACh-induced vasorelaxation enhanced from $44.4 \%$ to $55.1 \%$ ). This beneficial effect of irisin was attenuated when SnPP, API-2/triciribine or Compound $\mathrm{C}$ were added (Fig. 3B).

\section{Effect of irisin on total NO production}

The NO levels in the aorta with or without PVAT were significantly reduced in the HFD mice $(P<0.05)$. However, irisin significantly enhanced NO production both with PVAT and without PVAT $(P<0.05)$. We found that NO production was markedly reduced in the aorta with PVAT compared with that without PVAT from HFD mice $(P<0.05)$. This reduction was not shown in the control and irisin group $(P<0.05$, Fig. 4A).

\section{Effects of irisin on the HO-1/adiponectin axis in PVAT}

To examine whether irisin upregulated HO-1/adiponectin axis in PVAT, protein levels were measured by Western blot. As shown in Fig. 5, HO-1 and adiponectin levels in PVAT from the HFD mice were reduced compared with those in the control mice $(P<0.05)$, which were both enhanced when treating with irisin $(P<0.05)$. In an ex vivo study, incubating the CM derived from PVAT of HFD mice with irisin also increased the proteins of HO-1 and adiponectin.

\section{Effects of irisin on PVAT oxidative stress and inflammation}

We observed an increase in superoxide anion production and TNF- $\alpha$ expression in the PVAT from the HFD mice compared with the control mice $(p<0.05)$. However, both superoxide anion production and TNF- $\alpha$ expression were decreased after irisin treatment $(p$ $<0.05$; Fig. 4B and Fig. 6A).

Effects of irisin on FNDC5 and UCP-1 gene expression in PVAT

To investigate whether irisin influenced the browning gene in PVAT, FNDC5, Cidea and UCP-1 gene expression levels were measured by RT-PCT. The results showed that treatment of HFD with irisin increased FNDC5, Cidea and UCP-1 expression in PVAT $(P<0.05)$ (Fig. 6). 
Fig. 6. Effect of irisin on TNF- $\alpha$ expression (A), FNCD5 (B), UCP-1(C) and Cidea (D) gene expression in PVAT. $* \mathrm{P}<0.05 \mathrm{vs}$ NC group; "P $<0.05$ vs HFD group. $\mathrm{n}=4-6$ in each group.

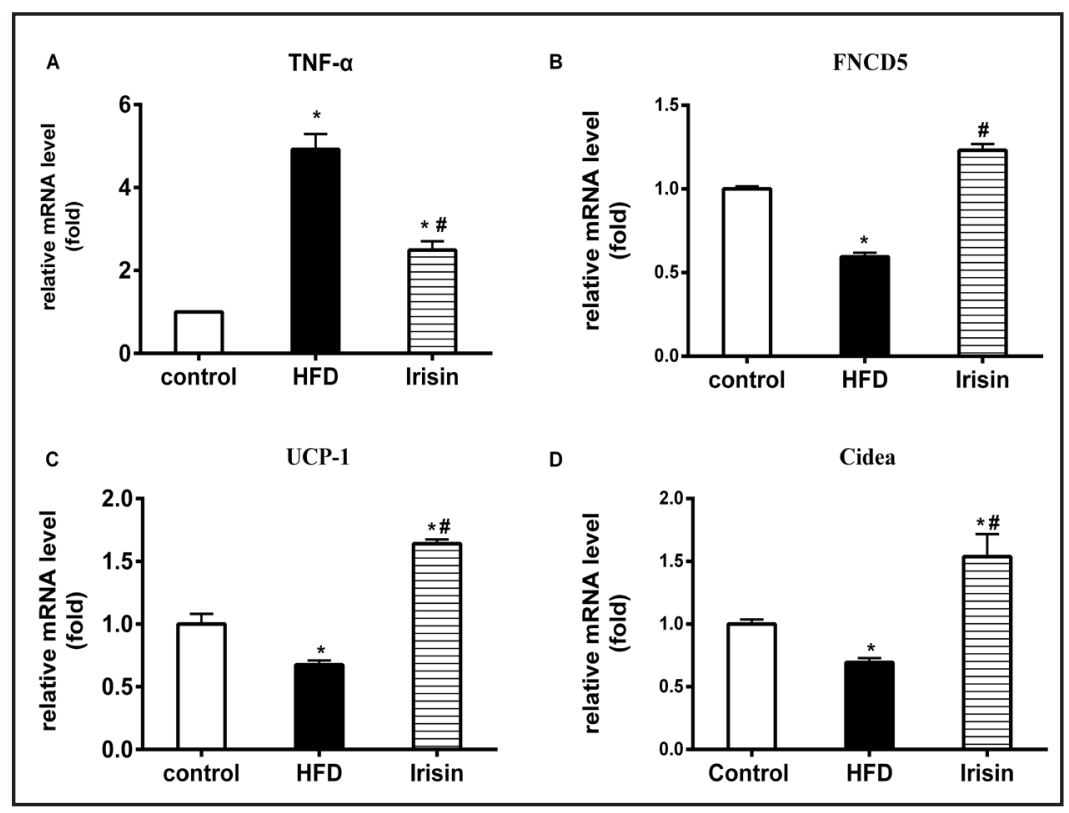

\section{Discussion}

We have demonstrated that irisin improves endothelial dysfunction in the aortas with PVAT from HFD-induced obese mice. This protective effect appears to be associated with upregulation of the HO-1/adiponectin axis in PVAT. These results further indicate a new mechanism that irisin plays in modulating endothelial function by regulating PVAT function in obesity.

The newly identified irisin, an exercise-induced myokine, secretes to circulation and mediates beneficial effects of exercise. It has been suggested that irisin seems to be beneficial for many metabolic diseases including diabetes and obesity through promoting the browning of white adipocytes [25]. Recent studies demonstrated that altered irisin levels are related to endothelial function [26, 27]. Irisin improves endothelial function and atherosclerosis in diabetic mice [27]. We previously found that irisin improved endothelial dysfunction in obese mice [12]. These studies indicate that irisin may play an important role in the regulation of endothelial function. However, most of the studies about endothelial function are concerned with the conditions of removed PVAT. With regard to PVAT that could exacerbate underlying endothelial dysfunction in obese subjects $[8,28]$, it is vital to know whether irisin also has a protective effect on endothelial function by regulating the PVAT function. Here, we demonstrated that treatment of HFD mice with irisin both in vivo and ex vivo significantly enhanced ACh-induced vasorelaxation in aortas with PVAT. To further investigate the direct effects of PVAT on endothelial function, we prepared CM by incubating PVAT from mice. As expected, incubated aortic rings (PVAT-) with CM (PVAT+) of HFD mice or CM (PVAT+) pretreated with PA impaired the EDV in control mice. However, this impairment was prevented by incubating the aortic rings (PVAT-) from HFD mice with CM (PVAT+) of irisin mice or with irisin ex vivo. These findings further indicate that irisin may play important roles in the protection against endothelial dysfunction by regulation of PVAT function.

Recently, many studies have demonstrated that induction of HO-1/adiponectin axis have beneficial effects on cardiovascular disease [16]. Both HO-1 and adiponectin have powerful anti-oxidative and anti-inflammatory properties that protect endothelial dysfunction by enhancing NO production [29-32]. In the current study, we found that irisin significantly increased HO-1 and adiponectin levels in PVAT from HFD mice and PVAT-derived CM, which is consistent with our previous study [12]. The beneficial effects of endothelial function by irisin-treated PVAT were partly weakened in the presence of inhibitors of HO-1/adiponectin. 
This indicates that HO-1/adiponectin axis in PVAT is involved in the regulation of endothelial function, and irisin could improve endothelial dysfunction by upregulating the HO-1/ adiponectin axis in PVAT.

Having demonstrated that irisin improves endothelial dysfunction by upregulating the HO-1/adiponectin axis in PVAT, we further investigated the underlying mechanisms responsible for this beneficial effect. As we know, endothelial function is mainly regulated by NO induced by eNOS phosphorylation. In our study, L-NAME, the eNOS inhibitor, could abolish all the vasorelaxation, indicating that Ach-dependent vasorelaxation is NO dependent. Treatment of HFD mice with irisin significantly increased NO production both with PVAT and without PVAT. We previously demonstrated that irisin may upregulate the HO-1/adiponectin axis and further activate AMPK-eNOS pathway to improve PVAT function through an endothelium-dependent pathway $[12,17]$. Upregulation of HO-1/adiponectin axis could also activate the AMPK-eNOS signaling pathway [16]. In addition, we also found that blocking the AMPK-eNOS pathway could abolish beneficial effects of irisin-treated PVAT on endothelial function. These findings further suggest that irisin could improve endothelial dysfunction via upregulation of the HO-1/adiponectin axis in PVAT and regulating the AMPKeNOS pathway.

Adipose tissue is an important mediator of oxidative stress and inflammation in obesity. PVAT, like other adipose tissues, also secrets adipokines and cytokines to regulate vascular function. Marchesi et al. [33] found that increased PVAT oxidative stress and inflammation lead to vascular dysfunction in obesity. PVAT-derived FFA, inflammatory cytokines and reactive oxygen species could impair endothelial function by reducing NO production and bioavailability. Our study found that after irisin treatment, not only circulating levels of FFA, TNF- $\alpha$, and MDA, but also PVAT superoxide production and TNF- $\alpha$ expression were decreased in HFD mice, which is consistent with our previous study [17]. Other studies have demonstrated that both HO-1 and adiponectin could prevent endothelial dysfunction by their powerful anti-oxidative and anti-inflammatory effects [32]. These protective effects of endothelial function may be associated with upregulation of the HO-1/adiponectin axis, and may indicate that irisin improves endothelial function by reducing local inflammation and oxidative stress in PVAT. However, whether irisin has direct anti-inflammatory and antioxidative effects independent of its beneficial metabolic effects should be clarified in the near future.

Another mechanism may account for this beneficial effect. PVAT is a unique adipose depot, displaying multiple characteristics of both white and brown adipose [34]. Our study showed that treatment of HFD with irisin increased FNDC5 gene expression, precursor of irisin, suggesting positive autoregulation of irisin in PVAT. And this further increased browning genes in PVAT from HFD mice, which shift the PVAT from white to brown characteristics. Browning of PVAT may upregulate HO-1 by PGC1 $\alpha$ and secret more protective cytokines such as adiponectin and less proinflammatory cytokines and, thus, may have a net beneficial effect on endothelial function [35].

The novelty of our research was to demonstrate that irisin improves endothelial function by modulating the HO-1/adiponectin axis in PVAT in obese mice. This study together with our previous studies demonstrate that irisin could improve function through PVAT-dependent or independent pathway $[12,17]$. There were some limitations in the current study. We did not use the control Ig G Fc peptide as the control for the experiments [36, 37]. And it will be optimal if we get the HFD group treated with Fc peptide. However, the recombinant irisin has been used in many studies, which showed that some beneficial effects were caused by irisin peptide. Future studies in our group should be needed to solve this problem. In addition, we did not use HO-1 and adiponectin knockout mice in the experiment. However, we used the inhibitors of HO-1/adiponectin to demonstrate that the beneficial effect of irisin was attenuated when inhibitors of HO-1/Adiponectin were present. And we believe it may not be optimal, but should draw a conclusion that irisin could improve endothelial dysfunction by upregulating the HO-1/adiponectin axis in PVAT. Further studies should be needed to confirm the specific mechanism.

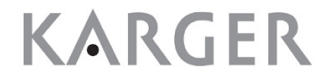




\section{Cellular Physiology Cell Physiol Biochem 2017;42:603-614 \begin{tabular}{l|l} 
and Biochemistry Publisned onIne: June 09, 2017 & $\begin{array}{l}\text { C } 2017 \text { The Author(s). Published by S. Karger AG, Basel } \\
\text { www.karger.com/cpb }\end{array}$ \\
\hline
\end{tabular}}

Hou et al.: Irisin Improves Endothelial Dysfunction

In summary, our work in vivo and ex vivo has demonstrated that irisin improved endothelial function by modulating the HO-1/adiponectin axis in PVAT in HFD-induced obese mice. These findings suggest that regulating PVAT function may be a potential target by which irisin improves endothelial function in obesity.

\section{Grants}

This work was supported by the National Natural Science Foundation of China (Grants NO. 81400829, 81600663 and 81300688), Natural Science Foundation of Shandong Province of China (Grants NO. ZR2015HL030), Medical Science and Technology Development Project of Shandong Province of China (Grants NO. 2016WS0678 and 2016WS0679) and Municipal Science and Technology Development Program of Weifang (Grants NO. 2016YX007).

\section{Disclosure Statement}

The authors declare no conflict of interest.

\section{References}

1 Chang L, Villacorta L, Li R, Hamblin M, Xu W, Dou C, Zhang J, Wu J, Zeng R, Chen YE: Loss of perivascular adipose tissue on peroxisome proliferator-activated receptor-gamma deletion in smooth muscle cells impairs intravascular thermoregulation and enhances atherosclerosis. Circulation 2012;126:1067-1078.

-2 Brown NK, Zhou Z, Zhang J, Zeng R, Wu J, Eitzman DT, Chen YE, Chang L: Perivascular adipose tissue in vascular function and disease: a review of current research and animal models. Arterioscler Thromb Vasc Biol 2014;34:1621-1630.

-3 Gao YJ, Lu C, Su LY, Sharma AM, Lee RM: Modulation of vascular function by perivascular adipose tissue: the role of endothelium and hydrogen peroxide. Br J Pharmacol 2007;151:323-331.

-4 Ouwens DM, Sell H, Greulich S, Eckel J: The role of epicardial and perivascular adipose tissue in the pathophysiology of cardiovascular disease. J Cell Mol Med 2010;14:2223-2234.

5 Gao YJ, Takemori K, Su LY, An WS, Lu C, Sharma AM, Lee RM: Perivascular adipose tissue promotes vasoconstriction: the role of superoxide anion. Cardiovasc Res 2006;71:363-373.

6 Bussey CE, Withers SB, Aldous RG, Edwards G, Heagerty AM: Obesity-Related Perivascular Adipose Tissue Damage Is Reversed by Sustained Weight Loss in the Rat. Arterioscler Thromb Vasc Biol 2016;36:13771385.

7 Sun X, Hou N, Han F, Guo Y, Hui Z, Du G, Zhang Y: Effect of high free fatty acids on the anti-contractile response of perivascular adipose tissue in rat aorta. J Mol Cell Cardiol 2013;63:169-174.

-8 Payne GA, Borbouse L, Kumar S, Neeb Z, Alloosh M, Sturek M, Tune JD: Epicardial perivascular adiposederived leptin exacerbates coronary endothelial dysfunction in metabolic syndrome via a protein kinase C-beta pathway. Arterioscler Thromb Vasc Biol 2010;30:1711-1717.

\9 Bostrom P, Wu J, Jedrychowski MP, Korde A, Ye L, Lo JC, Rasbach KA, Bostrom EA, Choi JH, Long JZ, Kajimura S, Zingaretti MC, Vind BF, Tu H, Cinti S, Hojlund K, Gygi SP, Spiegelman BM: A PGC1-alphadependent myokine that drives brown-fat-like development of white fat and thermogenesis. Nature 2012;481:463-468.

10 Zhu D, Wang H, Zhang J, Zhang X, Xin C, Zhang F, Lee Y, Zhang L, Lian K, Yan W, Ma X, Liu Y, Tao L: Irisin improves endothelial function in type 2 diabetes through reducing oxidative/nitrative stresses. J Mol Cell Cardiol 2015;87:138-147.

11 Hou N, Han F, Sun X: The relationship between circulating irisin levels and endothelial function in lean and obese subjects. Clin Endocrinol (Oxf) 2015;83:339-343.

12 Han F, Zhang S, Hou N, Wang D, Sun X: Irisin improves endothelial function in obese mice through the AMPK-eNOS pathway. Am J Physiol Heart Circ Physiol 2015;309:H1501-1508. 


\section{Cellular Physiology Cell Physiol Biochem 2017;42:603-614 \begin{tabular}{ll|l} 
and Biochemistry Published onlIne: June U9, 2017 & $\begin{array}{l}\text { (c) } 2017 \text { The Author(s). Published by S. Karger AG, Basel } \\
\text { www.karger.com/cpb }\end{array}$
\end{tabular}}

Hou et al.: Irisin Improves Endothelial Dysfunction

13 Li M, Kim DH, Tsenovoy PL, Peterson SJ, Rezzani R, Rodella LF, Aronow WS, Ikehara S, Abraham NG: Treatment of obese diabetic mice with a heme oxygenase inducer reduces visceral and subcutaneous adiposity, increases adiponectin levels, and improves insulin sensitivity and glucose tolerance. Diabetes 2008;57:1526-1535.

14 Liu X, Zang P, Han F, Hou N, Sun X: Renal protective effects of induction of haem oxygenase-1 combined with increased adiponectin on the glomerular vascular endothelial growth factor-nitric oxide axis in obese rats. Exp Physiol 2015;100:865-876.

15 Han F, Hui Z, Zhang S, Hou N, Wang Y, Sun X: Induction of haemeoxygenase-1 improves FFA-induced endothelial dysfunction in rat aorta. Cell Physiol Biochem 2015;35:1230-1240.

-16 Han F, Guo Y, Xu L, Hou N, Han F, Sun X: Induction of Haemeoxygenase-1 Directly Improves Endothelial Function in Isolated Aortas from Obese Rats through the Ampk-Pi3k/Akt-Enos Pathway. Cell Physiol Biochem 2015;36:1480-1490.

17 Hou N, Liu Y, Han F, Wang D, Hou X, Hou S, Sun X: Irisin improves perivascular adipose tissue dysfunction via regulation of the heme oxygenase-1/adiponectin axis in diet-induced obese mice. J Mol Cell Cardiol 2016;99:188-196.

18 Hou N, Han F, Wang M, Huang N, Zhao J, Liu X, Sun X: Perirenal fat associated with microalbuminuria in obese rats. Int Urol Nephrol 2014;46:839-845.

19 Li H, Wang YP, Zhang LN, Tian G: Perivascular adipose tissue-derived leptin promotes vascular smooth muscle cell phenotypic switching via p38 mitogen-activated protein kinase in metabolic syndrome rats. Exp Biol Med (Maywood) 2014;239:954-965.

20 Sun X, Yu Y, Han L: High FFA levels related to microalbuminuria and uncoupling of VEGF-NO axis in obese rats. Int Urol Nephrol 2013;45:1197-1207.

-21 Fleenor BS, Eng JS, Sindler AL, Pham BT, Kloor JD, Seals DR: Superoxide signaling in perivascular adipose tissue promotes age-related artery stiffness. Aging Cell 2014;13:576-578.

-22 Moon HS, Mantzoros CS: Regulation of cell proliferation and malignant potential by irisin in endometrial, colon, thyroid and esophageal cancer cell lines. Metabolism 2014;63:188-193.

-23 Li R, Wang WQ, Zhang H, Yang X, Fan Q, Christopher TA, Lopez BL, Tao L, Goldstein BJ, Gao F, Ma XL: Adiponectin improves endothelial function in hyperlipidemic rats by reducing oxidative/nitrative stress and differential regulation of eNOS/iNOS activity. Am J Physiol Endocrinol Metab 2007;293:E1703-1708.

24 Fitzgibbons TP, Kogan S, Aouadi M, Hendricks GM, Straubhaar J, Czech MP: Similarity of mouse perivascular and brown adipose tissues and their resistance to diet-induced inflammation. Am J Physiol Heart Circ Physiol 2011;301:H1425-1437.

25 Hofmann T, Elbelt U, Stengel A: Irisin as a muscle-derived hormone stimulating thermogenesis--a critical update. Peptides 2014;54:89-100.

-26 Zhang Y, Mu Q, Zhou Z, Song H, Zhang Y, Wu F, Jiang M, Wang F, Zhang W, Li L, Shao L, Wang X, Li S, Yang L, Wu Q, Zhang M, Tang D: Protective Effect of Irisin on Atherosclerosis via Suppressing Oxidized Low Density Lipoprotein Induced Vascular Inflammation and Endothelial Dysfunction. PLOS ONE 2016;11:e0158038.

27 Lu J, Xiang G, Liu M, Mei W, Xiang L, Dong J: Irisin protects against endothelial injury and ameliorates atherosclerosis in apolipoprotein E-Null diabetic mice. Atherosclerosis 2015;243:438-448.

-28 Ketonen J, Shi J, Martonen E, Mervaala E: Periadventitial adipose tissue promotes endothelial dysfunction via oxidative stress in diet-induced obese C57Bl/6 mice. Circ J 2010;74:1479-1487.

-29 Mandal P, Park PH, McMullen MR, Pratt BT, Nagy LE: The anti-inflammatory effects of adiponectin are mediated via a heme oxygenase-1-dependent pathway in rat Kupffer cells. Hepatology 2010;51:1420-1429.

-30 Nagano K, Ishida J, Unno M, Matsukura T, Fukamizu A: Apelin elevates blood pressure in ICR mice with LNAMEinduced endothelial dysfunction. Mol Med Rep 2013;7:1371-1375.

-31 Cheng CF, Lian WS, Chen SH, Lai PF, Li HF, Lan YF, Cheng WT, Lin H: Protective effects of adiponectin against renal ischemia-reperfusion injury via prostacyclin-PPARalpha-heme oxygenase-1 signaling pathway. J Cell Physiol 2012;227:239-249.

- 32 Abraham NG, Rezzani R, Rodella L, Kruger A, Taller D, Li VG, Goodman AI, Kappas A: Overexpression of human heme oxygenase-1 attenuates endothelial cell sloughing in experimental diabetes. Am J Physiol Heart Circ Physiol 2004;287:H2468-2477.

-33 Marchesi C, Ebrahimian T, Angulo O, Paradis P, Schiffrin EL: Endothelial nitric oxide synthase uncoupling and perivascular adipose oxidative stress and inflammation contribute to vascular dysfunction in a rodent model of metabolic syndrome. Hypertension 2009;54:1384-1392. 


\section{Cellular Physiology Cell Physiol Biochem 2017;42:603-614}

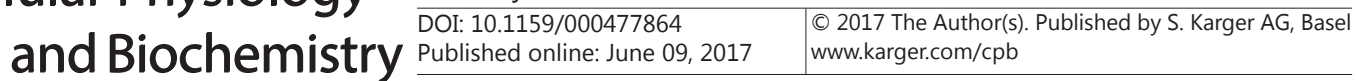

Hou et al.: Irisin Improves Endothelial Dysfunction

-34 Padilla J, Jenkins NT, Vieira-Potter VJ, Laughlin MH: Divergent phenotype of rat thoracic and abdominal perivascular adipose tissues. Am J Physiol Regul Integr Comp Physiol 2013;304:R543-552.

-35 Singh SP, Schragenheim J, Cao J, Falck JR, Abraham NG, Bellner L: PGC-1 alpha regulates HO-1 expression, mitochondrial dynamics and biogenesis: Role of epoxyeicosatrienoic acid. Prostaglandins Other Lipid Mediat 2016;125:8-18.

36 Zhang W, Chang L, Zhang C, Zhang R, Li Z, Chai B, Li J, Chen E, Mulholland M: Irisin: A myokine with locomotor activity. Neurosci Lett 2015;595:7-11.

37 Zhang W, Chang L, Zhang C, Zhang R, Li Z, Chai B, Li J, Chen E, Mulholland M: Central and peripheral irisin differentially regulate blood pressure. Cardiovasc Drugs Ther 2015;29:121-127. 